\title{
Total internal reflection imaging ellipsometry biosensor: Its principle and applications
}

Cite as: AIP Conference Proceedings 2110, 020007 (2019); https://doi.org/10.1063/1.5110801

Published Online: 07 June 2019

Ting Wang, Ke Ma, Wei Liu, Gang Jin, and Yu Niu

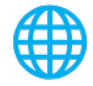

\section{ARTICLES YOU MAY BE INTERESTED IN}

Optimization of temperature shift-based fermentation process for sophorolipids production using response surface methodology

AIP Conference Proceedings 2110, 020002 (2019); https://doi.org/10.1063/1.5110796

Preface: International Symposium on the Frontiers of Biotechnology and Bioengineering (FBB 2019)

AIP Conference Proceedings 2110, 010001 (2019); https://doi.org/10.1063/1.5110794

Research on the intelligent pharmacy construction of Chinese herbal pieces

AIP Conference Proceedings 2110, 020005 (2019); https://doi.org/10.1063/1.5110799

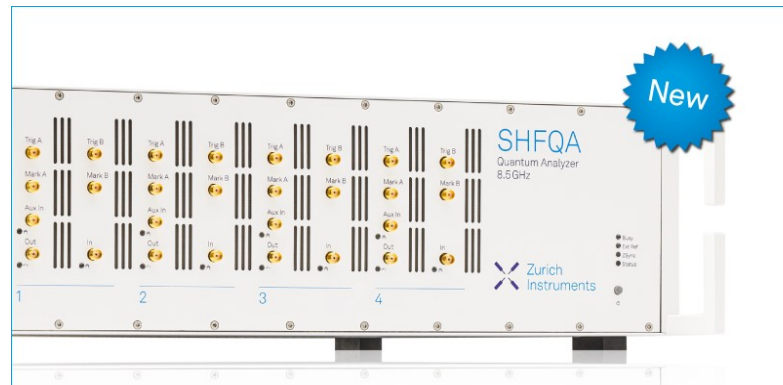

Your Qubits. Measured.

Meet the next generation of quantum analyzers

- Readout for up to 64 qubits

- Operation at up to $8.5 \mathrm{GHz}$.

Find out more mixer-calibration-free

- Signal optimization with minimal latency

Zurich

Instruments 


\title{
Total Internal Reflection Imaging Ellipsometry Biosensor: Its Principle and Applications
}

\author{
Ting Wang ${ }^{1,2, b)}$, Ke $\mathrm{Ma}^{1,2, b)}$, Wei Liu' ${ }^{2)}$, Gang Jin ${ }^{1,2)}$ and $\mathrm{Yu} \mathrm{Niu}^{2, \text { a) }}$ \\ ${ }^{1}$ School of Engineering Sciences, University of Chinese Academy of Sciences, Beijing 100049, China. \\ ${ }^{2} N M L$, Beijing Key Laboratory of Engineered Construction and Mechanobiology, Institute of Mechanics, Chinese \\ Academy of Sciences, Beijing 100190, China.

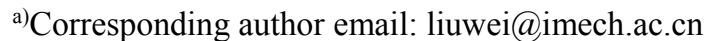 \\ b) These authors contributed equally to this work
}

\begin{abstract}
After years of development, total internal reflection imaging ellipsometry (TIRIE) biosensor has advanced from a concept to a practical analysis tool for biomolecule interactions. Compared to the traditional biosensor with imaging ellipsometry (BIE) which works under the external reflection condition, not only does TIRIE biosensor perform the labelfree, high throughput detection, but also remains competitive detection sensitivity. The review summarizes the principle, the equipment setup, and the current applications of TIRIE biosensor.
\end{abstract}

Key words: Imaging Ellipsometry; Surface Plasmon Resonance; Evanescent Wave; Total Internal Reflection; Label-free biosensor.

\section{INTRODUCTION}

A biosensor, which commonly integrates a biological component with a physicochemical transduction, has been used as an analytical device for certain bio-material sample analysis [1-3]. In general, the biological component plays a central role in analyte recognition, while the physicochemical transduction transforms the recognition into a physics detectable signal [2]. To meet the immense demand of detecting various biomolecule analytes in practice, numerous biosensors have been developed rapidly [4-7] and emerged in industry for the past few decades [8-13].

By detection mode, these biosensors can be classified into two categories: one introducing the labeling compounds [14-19] and the other employing label-free method [20-24]. The typical labeling methods in biosensors include fluorescence[17, 18], enzyme[15], radioactivity[19] and the more recently developed quantum dot[25]. Depending on the amplification of the labeling, the labeling biosensors have achieved the impressive detection limit, sensitivity and specificity in biomolecule interaction analysis. However, the introduction of the labeling compounds inevitably gives rise to some drawbacks. First, the labeling may change the biomolecule conformations and thus impair their bioactivity[26]. Furthermore, it is difficult to perform quantitative detection owing to the inhomogeneity introduced by the labeling[19]. In addition, the labeling process usually time consuming and labor intensive. To solve these problems, label-free biosensors are the alternative to detect the biomolecule interactions which are fundamentally inaccessible to the labeling method. The label-free biosensors mainly rely on optics[24], acoustics[27], quartz crystal resonators[28], nanowires[29], ion channel switch[30] and notably surface plasmon resonance (SPR)[31, 32]. However, it is still a challenge to achieve high-throughput and great sensitivity at the same time [33, 34].

To perform high throughput detection with great sensitivity, we have developed an imaging ellipsometry system, which works under the external reflection condition and uses the conventional polarizer-compensator-sample-analyzer (PCSA) configuration and a charge coupled device (CCD) camera to get the images of the sample[35]. As the application of the system, we use it as a biosensor based on imaging ellipsometry (BIE) to perform the label-free detection of the biomolecule interactions on the substrate [36-38]. We modify the antigen on the silicon based substrate 
and when we submerge the chip into the solution contains the corresponding antibody, the thickness of the biomolecules on chip will increase and by the imaging ellipsometry, we could deduce the thickness and get the information of such interactions between the antigen and the antibody as tumor markers of CD146[39], avian influenza virus subtype H5 hepatitis B virus[40] and Tropomyosin Allergens[41] with the sub-nanometer accuracy. Furthermore, by the imaging technique, the biosensor enjoys high-throughput capability, which makes it perform multi-sample analysis with ease.

Hoping to get the kinetics of the biomolecule interactions on the substrate, we invented a cell for real-time detection [42]. As an application, we use it to observe the competitive adsorption of collagen/ bovine serum albumin (BSA) on the hydrophobic and hydrophilic substrates [43].

However, there are some problems in the system. First, the sample consumption is quite large, which makes the detection for some rare protein rather expensive, sometimes, not affordable. A second problem is the solution disturbance limit the sensitivity of the system. For example, the detection limit of BSA, is 0.1 milligram per milliliter, far higher than the concentration of the interested protein in human serum. Thus, to improve the sensitivity is the key to the system.

To improve the sensitivity, total internal reflection imaging ellipsometry (TIRIE) biosensor has been developed for decades[44]. In this review, we summarize the principle, the equipment setup, the detection procedure, and the current applications of TIRIE biosensor.

\section{THE PRINCIPLE OF IMAGING ELLIPSOMETRY WORKS UNDER THE TOTAL INTERNAL REFLECTION CONDITION}

At the beginning, we will discuss the principle of imaging ellipsometry works under the total internal reflection condition briefly. We will start from the internal reflection for a PCSA system at the interface between two dielectric mediums for it cannot only give us the basic physics of the picture but the simple mathematics. The theory is classical for such system: Fresnel law and Snell's law, which can be found in many textbooks. Then, the surface enhancement effect by the gold film will be introduced.

\section{Internal Reflection at the Interface between Two Dielectric Medium}

To evaluate the imaging system, the relative imaging contrast is defined as the image contrast caused by the protein adsorption and compared it with the original substrate at which no protein adsorption occurs:

$$
\frac{\delta I}{I}=\frac{I\left(\phi_{0}, P, A, d_{p} r\right)-I\left(\phi_{0}, P, A, 0\right)}{I\left(\phi_{0}, P, A, 0\right)}
$$

$\frac{\delta \mathrm{I}}{\mathrm{I}}=\frac{\mathrm{I}\left(\phi_{0}, \mathrm{P}, \mathrm{A}, \mathrm{d}_{\mathrm{p}} \mathrm{r}\right)-\mathrm{I}\left(\phi_{0}, \mathrm{P}, \mathrm{A}, 0\right)}{\mathrm{I}\left(\phi_{0}, \mathrm{P}, \mathrm{A}, 0\right)}$ Where $\phi_{0}$ is the angle of incidence, $\mathrm{P}$ is the azimuth setting of the polarizer, $\mathrm{A}$ is the azimuth setting of the analyzer, $d_{p} r$ is the thickness of the adsorbed protein layer.

For the internal reflection at the interface between the two dielectric mediums, two specific angles exist in the system. The first one is Brewster angle, around $37.7^{\circ}$ in our system. However, because the relative imaging contrast decreases sharply around Brewster angle, the application is not as promising as it indicates in Fig. 1a. The other angle is the critical angle. Owing to the total internal reflection, there is no imaging contrast at all.

However, there is still a transmission field, so-called evanescent wave, under the total internal reflection condition, which travels along the interface and its energy decays exponentially into the solution. One of the interesting property about the evanescent wave is that its amplitude can be larger than that of the incident light, 7 times higher for ppolarized wave[45]. Because of the evanescent wave, the reflection will carry the information of the interface. That is the polarization of the reflection will be modulated by the interface.

Thus, by introducing the polarization around the total internal reflection condition, we could get the imaging contrast. Although the relative change is still subtle, compared with the situation with no polarization, 100 times enhancement can be achieved, as shown in Fig. 1b. However, it is still insufficient for the practical detection. 


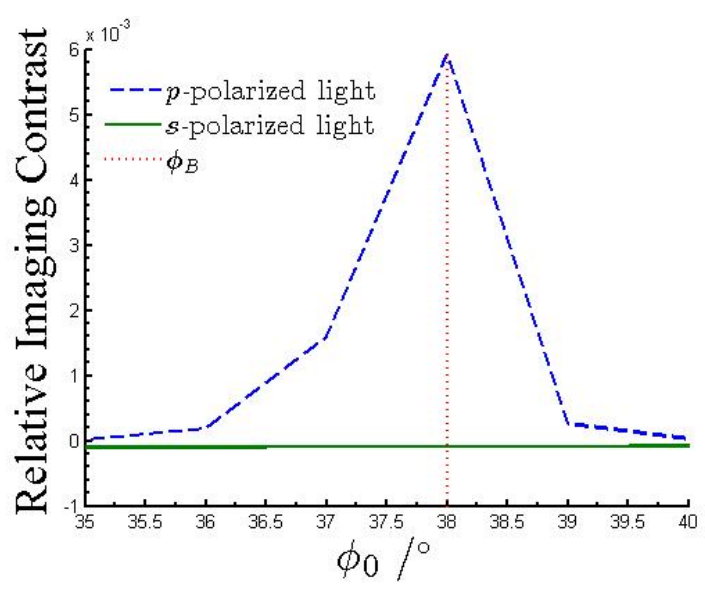

(a)

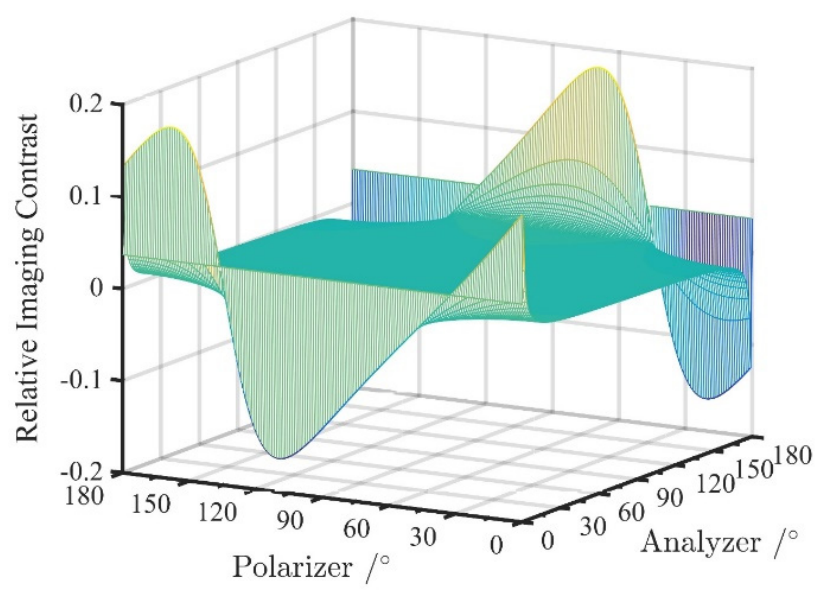

(b)

FIGURE 1. For the internal reflection at the interface between the two dielectric mediums, the relative imaging contrast from 1 $\mathrm{nm}$ protein adsorption around the Brewster angle. The refractive index of the glass, the protein adsorption layer, and the liquid are $1.72,1.46,1.33$, respectively. At $\phi_{B} \approx 37.7^{\circ}, \psi \approx 0.5^{\circ}, \Delta=180^{\circ}$. (a) without polarization, (b) with polarization. The relative imaging contrast reaches maximum at $A=\psi \approx 0.5^{\circ}$ and $P \approx 45^{\circ}$.

\section{Surface Enhancement Effect by Gold Film}

To improve the imaging contrast further, a layer of gold has been introduced into the glass/solution interface. Thus, the basic model can be described as glass/gold/protein/liquid and Fresnel law and Snells law still hold.

As is shown in Fig. 2a, after we introduce the gold layer on the surface, 10000 times improvement has been achieved at the optimized angle, around $58^{\circ}$, and the optimized gold thickness, about $50 \mathrm{~nm}$ thick gold, which is known as SPR effect. In Fig. 2b, a polarizer and an analyzer have been introduced to perform polarization effect, by the optimized polarization settings at the incident angle $58^{\circ}$, another 10 times improvement has been achieved. 


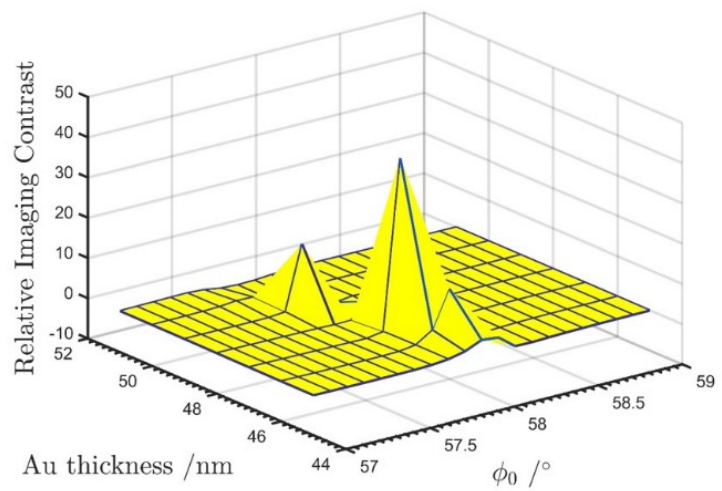

(a)

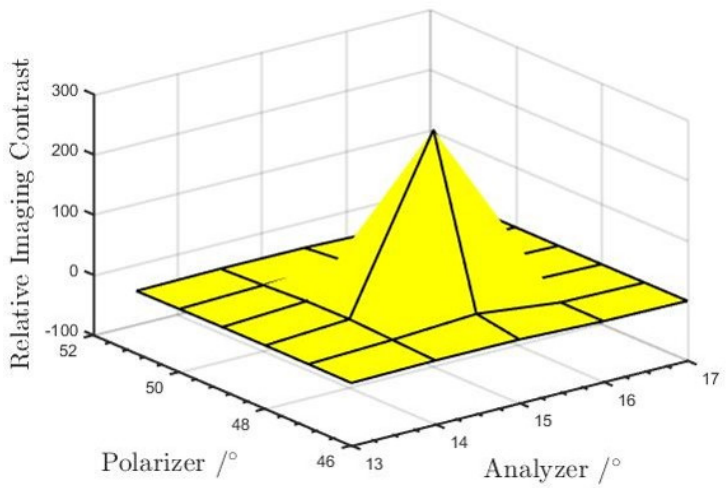

(b)

FIGURE 2. (a) The relative imaging contrast of TIRIE system without polarization varies with the angle of incidence and the thickness of the gold layer when $1 \mathrm{~nm}$ protein has adsorbed at the glass/gold/buffer interface. The refractive index of the glass, the gold, the protein and the buffer are $1.72,0.18-3.43 \mathrm{j}, 1.46$ and 1.33 respectively; (b) the relative imaging contrast of TIRIE system with polarization at the glass $/ 50 \mathrm{~nm}$ gold/buffer interface at $\phi_{0}=57^{\circ}$ when $\psi \approx 15^{\circ}$ and $\Delta \approx 170^{\circ}$. The largest response is achieved at the optimized setting, $A=\psi \approx 15^{\circ}, P \approx 50^{\circ}$.

\section{TIRIE BIOSENSOR SETUP AND THE GENERAL DETECTION PROCEDURE}

The schematic illustration of TIRIE biosensor in our lab is shown in Fig. 3 and it is mainly composed of an imaging ellipsometer operated under the total internal reflection mode and a micro-fluidic reactor array with 24 independent reaction cells. A Xe lamp is used as the light source and a high-speed cool CCD camera as the detector. For most applications, the $630 \mathrm{~nm}$ light beam is guided by an optical fiber and expanded by a collimating system. A SF10 trapezoidal prism is used to couple the light beam with the gold covered sensing surface. After passing a polarizer and a compensator, the polarized collimated beam propagates perpendicularly to the prism and onto the sensing surface. When the incident angle is $58^{\circ}$, in the neighborhood of SPR angle for the system, the evanescent wave appears sharply at the sensing surface to detect the interaction in the depth of $200 \mathrm{~nm}$ from the surface. The reflected light carrying the surface information is then imaged by a CCD camera after passing an analyzer. To achieve the best sensitivity, the system works under the off-null mode. During the measurement, the optical signal variation from the biomolecule adsorption is recorded by CCD in grayscale. 


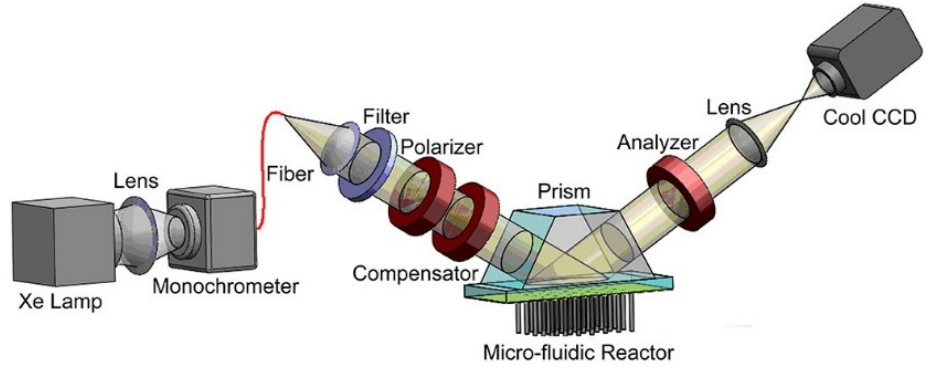

FIGURE 3. Schematic diagram of TIRIE biosensor[46].

The general procedure to detect the biomolecule interaction by TIRIE is demonstrated in Fig. 4. Generally, a ligand and its receptor (also called analyte), such as an antibody and its corresponding antigen, are assembled at the sensing surface to form a bio-complex due to their affinity. Firstly, the ligand is immobilized to the substrate to form a bioprobe sensing surface. After the surface rinsing and the blocking, the analyte is pumped into the micro-fluidic reactor. Then, the analyte will interact with its corresponding ligand to form a bio-complex due to their specific bio-affinity, which will vary the surface coverage. The whole process will be recorded by TIRIE. A general procedure for TIRIE biosensor to detect biomolecule interactions is illustrated in Fig. 4.
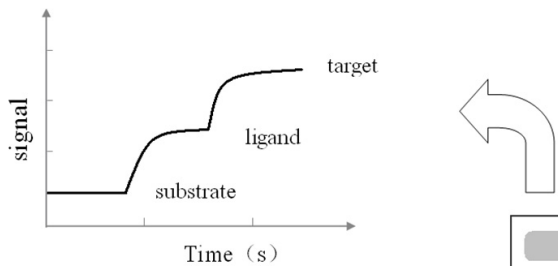

Time $(s)$
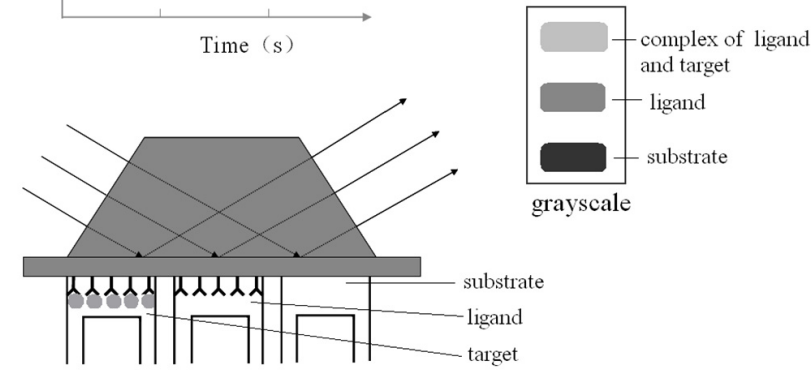

FIGURE 4. The general procedure for TIRIE biosensor to detect biomolecule interactions[46].

\section{CURRENT APPLICATIONS OF TIRIE BIOSENSOR}

TIRIE biosensor has been exploited several representative applications in practice, including the quantitative serological detection of tumor marker CA 19-9[47], the observation of weak interaction between tris and lysozyme[48], the characterization of vesicle adsorption on gold surface and the quantitative detection of dissolved oxygen in water[49, 50].

A highly sensitive quantitative detection of tumor marker CA 19-9 has been performed by TIRIE biosensor. As shown in Fig. 5, protein A is immobilized on the PEGylated phospholipid membrane constructed on TIRIE biosensor substrate, and then CA 19-9 antibody is assembled to form the biosensing surface. Since the PEGylated lipid membrane can resistant strongly against non-specific adsorption, serological detection of CA 19-9 can be carried out without the previous blocking process. Dose-response calibration curve is established for CA 19-9 quantitative detection. The liner detection range is from $10 \mathrm{U} / \mathrm{mL}$ to $1000 \mathrm{U} / \mathrm{mL}$ and the limit of detection reaches $18.2 \mathrm{U} / \mathrm{mL}$, both of which meets the requirement of the clinic detection. 15 serum samples have been detected and their results are compared with those of electro-chemiluminescence method as a commercial immunoassay. By the regression analysis, the results from the two methods present good consistence, exhibiting the potential application in early cancer screening of TIRIE biosensor. 
(a)

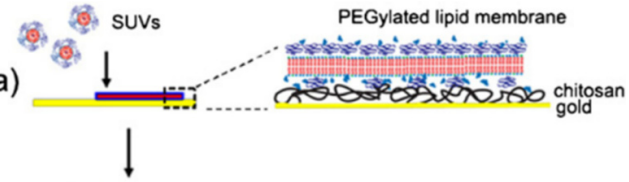

(b)

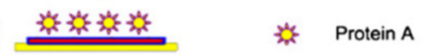

(c)
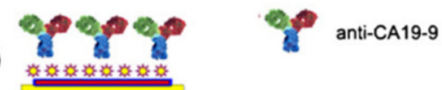

(d)

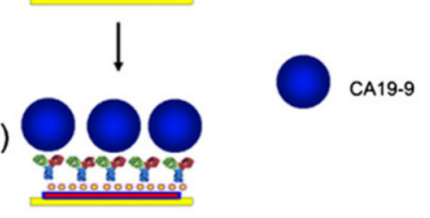

FIGURE 5. Flow chart of CA19-9 detection on PEGylated phospholipid membrane with TIRIE biosensor. (a) Phospholipid membrane formation through SUVs; (b) protein A coupling on phospholipid membrane; (c) antibody attachment; (d) CA19-9 binding[47].

Weak affinity interactions among biomolecules have attracted more and more attentions for they play a significant role in organisms. However, since weak affinity interactions are mainly formed by hydrogen bonds and van del Waals interactions, their binding complexes are usually transient and unstable. Thus, it is difficult to characterize these interactions due to the limitation of the detection approaches. The interaction between tris and lysozyme (LZM), a typical example of weak affinity interactions, has been detected with TIRIE biosensor as a trial. Tris is immobilized on the gold thin film substrate to form the biosensing surface and then LZM as well as its negative controls is delivered to the tris-immobilized surface. The interaction process between tris and LZM is recorded by TIRIE biosensor to form a real-time curve (Fig. 6) and its dissociation constant is deduced as $7.6 \times 10^{-5} \mathrm{M}$, which is in agreement with the results in the reported work by other investigators. The results indicate that TIRIE biosensor is competent for weak affinity interaction analysis.

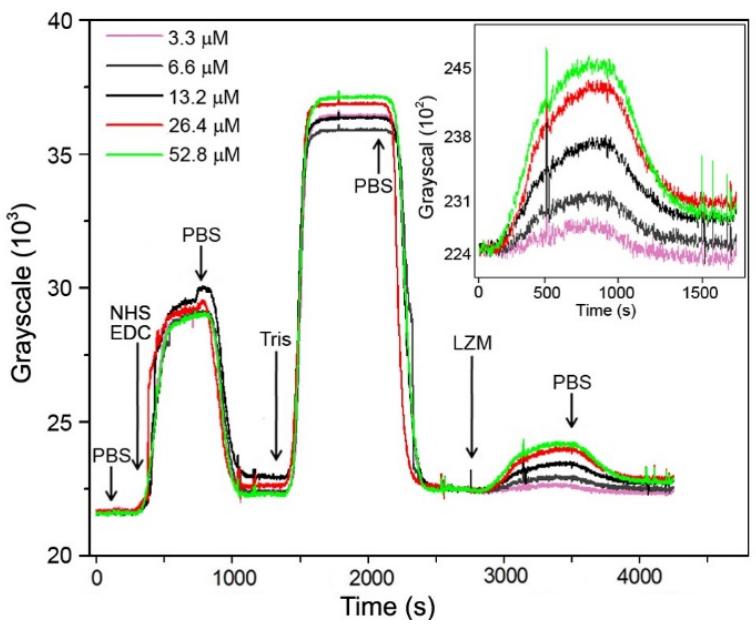

FIGURE 6. The real-time curve for the interaction between tris and LZM at different concentrations. LZM is diluted to the concentrations of $3.3,6.6,13.2,26.4$ and $52.8 \mu \mathrm{mol} / \mathrm{L}$ and then delivered to the tris immobilized surface. The inset in the figure is clearly to show the specific binding process between tris and LZM[51].

The applications of TIRIE biosensor are not limited to biomolecule interaction but can be extended to other fields. Combined with the electrochemistry unit, electrochemistry- TIRIE (EC-TIRIE) has been developed. Fig. 7a illustrates the EC-TIRE system. EC-TIRIE shown its strength in water environment detection. Since dissolved oxygen (DO) in water plays an important role in biochemical oxygen demand. EC-TIRIE is used to observe the DO reduction at the 
sensing surface. It can tell the DO concentration from $2 \mathrm{mg} / \mathrm{L}$ to $8 \mathrm{mg} / \mathrm{L}$ (Fig. 7b). By introducing amplification medium, such as surface tethered weak polyelectrolyte, polyaniline with Iron-porphyrin, the detection limit of DO has improved 10 times.

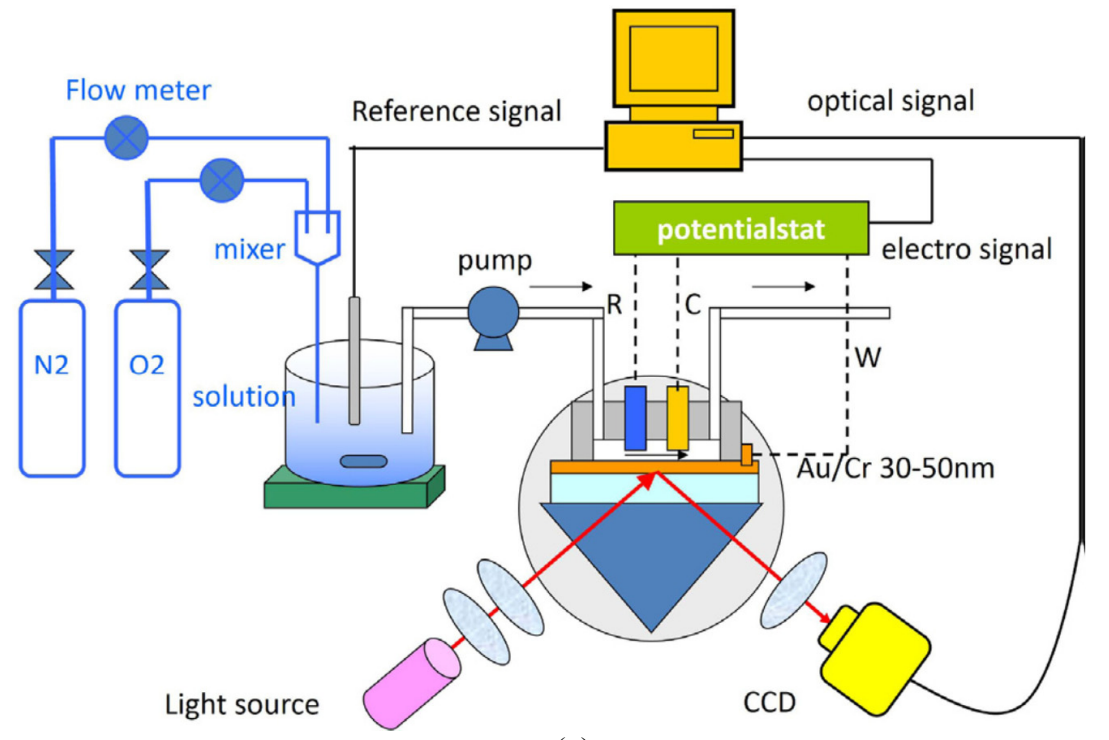

(a)

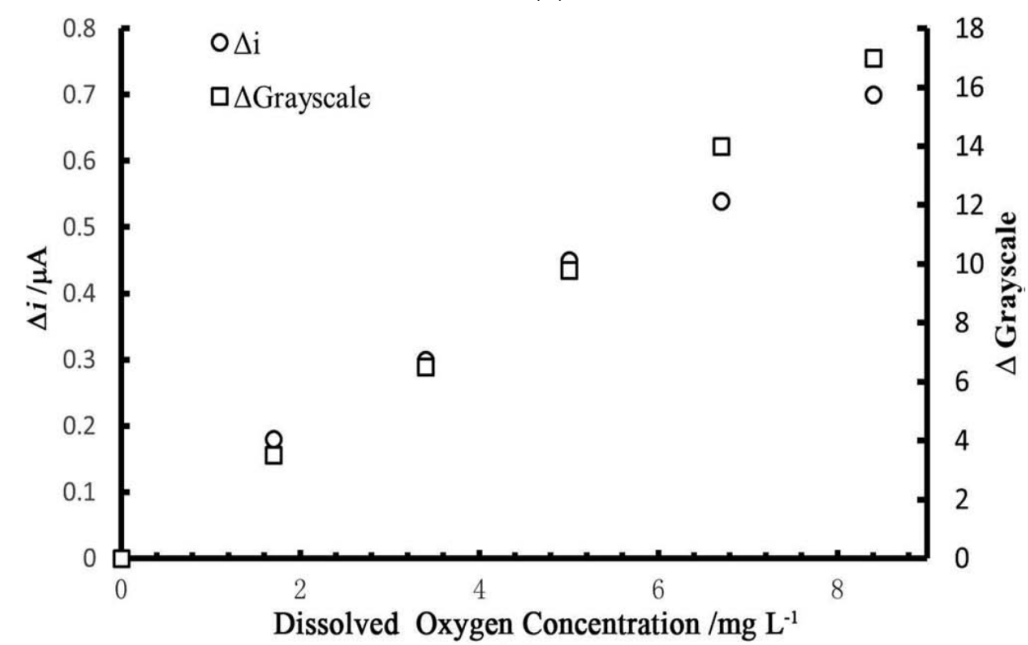

(b)

FIGURE 7. (a) Schematic illustration of EC-TIRIE system [49]. (b) The dissolved oxygen calibration curves show that both electric signal change and TIRIE signal change are proportional to the DO concentration [50].

\section{CONCLUSIONS AND PERSPECTIVE}

Combined the imaging ellipsometry with SPR phenomenon, TIRIE has been successfully applied to perform realtime, high-throughput, and sensitive detection in biomolecule interaction analysis and pollution monitoring. Although ellipsometry technique can guarantee the subnanometer accuracy, there is still the possibility to improve the signal noise ratio (SNR). One approach is to introduce the meta-materials at the sensing surface. However, it is seldomly reported to realize the high-throughput detections on the meta-surface. The other approach is to supress the noise of the system. Currently, the noise source of TIRIE system comes from the light source instability and temperature fluctuation. By our estimation, the SNR will be improved at least 10 times after the noise has been deliberately suppressed. 


\section{ACKNOWLEDGMENTS}

The authors thank the financial support to the National Basic Research Program of China (2015CB352100), to the National Natural Science Foundation of China (81872584 and 21305147), to the Science and Technology Service Network Initiative of the Chinese Academy of Sciences (KFJ-STS-QYZD-012), to the Youth Innovation Promotion Association of the Chinese Academy of Sciences (2017026), and to the International Science \& Technology Cooperation Program of China (2015DFG32390).

\section{REFERENCES}

1. Homola, J., Surface plasmon resonance sensors for detection of chemical and biological species. Chemical Reviews, 2008. 108(2): p. 462-493.

2. Su, L.A., et al., Microbial biosensors: A review. Biosensors \& Bioelectronics, 2011. 26(5): p. 1788-1799.

3. Xu, X. and Y.B. Ying, Microbial Biosensors for Environmental Monitoring and Food Analysis. Food Reviews International, 2011. 27(3): p. 300-329.

4. Albers, J., et al., Electrical biochip technology - a tool for microarrays and continuous monitoring. Analytical and Bioanalytical Chemistry, 2003. 377(3): p. 521-527.

5. Venkatasubbarao, S., Microarrays - status and prospects. Trends in Biotechnology, 2004. 22(12): p. $630-637$.

6. Vo-Dinh, T., B.M. Cullum, and D.L. Stokes, Nanosensors and biochips: frontiers in biomolecular diagnostics. Sensors and Actuators B-Chemical, 2001. 74(1-3): p. 2-11.

7. Cheng, M.M.C., et al., Nanotechnologies for biomolecular detection and medical diagnostics. Current Opinion in Chemical Biology, 2006. 10(1): p. 11-19.

8. Jain, K.K., Applications of biochips: From diagnostics to personalized medicine. Current Opinion in Drug Discovery \& Development, 2004. 7(3): p. 285-289.

9. Ramachandran, N., S. Srivastava, and J. LaBaer, Applications of protein microarrays for biomarker discovery. Proteomics Clinical Applications, 2008. 2(10-11): p. 1444-1459.

10. Wiesner, A., Detection of tumor markers with ProteinChip $((\mathrm{R}))$ technology. Current Pharmaceutical Biotechnology, 2004. 5(1): p. 45-67.

11. FitzGerald, S.P., et al., Development of a high-throughput automated analyzer using biochip array technology. Clinical Chemistry, 2005. 51(7): p. 1165-1176.

12. Prix, L., et al., Diagnostic biochip array for fast and sensitive detection of K-ras mutations in stool. Clinical Chemistry, 2002. 48(3): p. 428-435.

13. Kim, D.S., et al., Disposable integrated microfluidic biochip for blood typing by plastic microinjection moulding. Lab on a Chip, 2006. 6(6): p. 794-802.

14. Huang, R.P., Protein arrays, an excellent tool in biomedical research. Frontiers in Bioscience-Landmark, 2003. 8: p. D559-D576.

15. Varnum, S.M., et al., Enzyme-amplified protein microarray and a fluidic renewable surface fluorescence immunoassay for botulinum neurotoxin detection using high-affinity recombinant antibodies. Analytica Chimica Acta, 2006. 570(2): p. 137-143.

16. Pickering, J.W., et al., A 22-plex chemiluminescent microarray for pneumococcal antibodies. American Journal of Clinical Pathology, 2007. 128(1): p. 23-31.

17. Ruano, J.M., et al., Design and fabrication of a silica on silicon integrated optical biochip as a fluorescence microarray platform. Biosensors \& Bioelectronics, 2003. 18(2-3): p. 175-184.

18. Shigaki, S., et al., A peptide microarray for the detection of protein kinase activity in cell lysate. Analytical Sciences, 2007. 23(3): p. 271-275.

19. Kodadek, T., Protein microarrays: prospects and problems. Chemistry \& Biology, 2001. 8(2): p. 105-115.

20. Hutchens, T.W. and T.T. Yip, NEW DESORPTION STRATEGIES FOR THE MASS-SPECTROMETRIC ANALYSIS OF MACROMOLECULES. Rapid Communications in Mass Spectrometry, 1993. 7(7): p. 576-580.

21. Martin, S.J., V.E. Granstaff, and G.C. Frye, CHARACTERIZATION OF A QUARTZ CRYSTAL MICROBALANCE WITH SIMULTANEOUS MASS AND LIQUID LOADING. Analytical Chemistry, 1991. 63(20): p. 2272-2281.

22. Rodahl, M., et al., QUARTZ-CRYSTAL MICROBALANCE SETUP FOR FREQUENCY AND Q-FACTOR MEASUREMENTS IN GASEOUS AND LIQUID ENVIRONMENTS. Review of Scientific Instruments, 1995. 66(7): p. 3924-3930. 
23. Ziegler, C., Cantilever-based biosensors. Analytical and Bioanalytical Chemistry, 2004. 379(7-8): p. 946-959.

24. Heitzer, A., et al., OPTICAL BIOSENSOR FOR ENVIRONMENTAL ONLINE MONITORING OF NAPHTHALENE AND SALICYLATE BIOAVAILABILITY WITH AN IMMOBILIZED BIOLUMINESCENT CATABOLIC REPORTER BACTERIUM. Applied and Environmental Microbiology, 1994. 60(5): p. 1487-1494.

25. Li, X., et al., Self-assembled graphene quantum dots induced by cytochrome c: a novel biosensor for trypsin with remarkable fluorescence enhancement. Nanoscale, 2013. 5(17): p. 7776-7779.

26. Dupuy, A.M., S. Lehmann, and J.P. Cristol, Protein biochip systems for the clinical laboratory. Clinical Chemistry and Laboratory Medicine, 2005. 43(12): p. 1291-1302.

27. Andle, J.C. and J.F. Vetelino, Acoustic wave biosensors, in 1995 Ieee Ultrasonics Symposium Proceedings, Vols 1 and 2, M. Levy, S.C. Schneider, and B.R. McAvoy, Editors. 1995. p. 451-460.

28. Evans, J.A., et al., Detection of protease activity on a quartz crystal biosensor. Faseb Journal, 2002. 16(5): p. A1194-A1194.

29. Yogeswaran, U. and S.-M. Chen, A review on the electrochemical sensors and biosensors composed of nanowires as sensing material. Sensors, 2008. 8(1): p. 290-313.

30. Krishnamurthy, V., S.M. Monfared, and B. Cornell, Ion Channel Biosensors-Part II: Dynamic Modeling, Analysis, and Statistical Signal Processing. Ieee Transactions on Nanotechnology, 2010. 9(3): p. 313-321.

31. Scarano, S., et al., Surface plasmon resonance imaging for affinity-based biosensors. Biosensors \& Bioelectronics, 2010. 25(5): p. 957-966.

32. Homola, J., S.S. Yee, and G. Gauglitz, Surface plasmon resonance sensors: review. Sensors and Actuators BChemical, 1999. 54(1-2): p. 3-15.

33. Westphal, P. and A. Bornmann, Biornolecular detection by surface plasmon enhanced ellipsometry. Sensors and Actuators B-Chemical, 2002. 84(2-3): p. 278-282.

34. Boozer, C., et al., Looking towards label-free biomolecular interaction analysis in a high-throughput format: a review of new surface plasmon resonance technologies. Current Opinion in Biotechnology, 2006. 17(4): p. 400405.

35. Jin, G., et al., A BIOSENSOR CONCEPT BASED ON IMAGING ELLIPSOMETRY FOR VISUALIZATION OF BIOMOLECULAR INTERACTIONS. Analytical Biochemistry, 1995. 232(1): p. 69-72.

36. Wang, Z.H. and G. Jin, A label-free multisensing immunosensor based on imaging ellipsometry. Analytical Chemistry, 2003. 75(22): p. 6119-6123.

37. Qi, C., et al., Investigation of interaction between two neutralizing monoclonal antibodies and SARS virus using biosensor based on imaging ellipsometry. Biomedical Microdevices, 2006. 8(3): p. 247-253.

38. Zhang, H.G., et al., Evaluation of a new CA15-3 protein assay method: Optical protein-chip system for clinical application. Clinical Chemistry, 2005. 51(6): p. 1038-1040.

39. Niu, Y., et al., Approach to Quantitative Detection of CD146 with the Label-free Protein Biosensor Based on Imaging Ellipsometry, in Sensors, Cameras, and Systems for Industrial, Scientific, and Consumer Applications Xii, R. Widenhorn and V. Nguyen, Editors. 2011, Spie-Int Soc Optical Engineering: Bellingham.

40. Qi, C., et al., Detection of avian influenza virus subtype H5 using a biosensor based on imaging ellipsometry. Biosensors \& Bioelectronics, 2010. 25(6): p. 1530-1534.

41. Wang, W., et al., Analysis of the interaction between tropomyosin allergens and antibodies using a biosensor based on imaging ellipsometry. Anal Chem, 2013. 85(9): p. 4446-52.

42. Wang, Z.H., et al., A label-free protein microfluidic array for parallel immunoassays. Electrophoresis, 2006. 27(20): p. 4078-4085.

43. Ying, P.Q., G. Jin, and Z.L. Tao, Competitive adsorption of collagen and bovine serum albumin - effect of the surface wettability. Colloids and Surfaces B-Biointerfaces, 2004. 33(3-4): p. 259-263.

44. Chen, Y., et al., Biosensor with total internal reflection imaging ellipsometry. International Journal of Nanotechnology, 2007. 4: p. 171-178.

45. Liu, L., et al., Optimization of evanescent wave imaging for the visualization of protein adsorption layers. Science China Physics, Mechanics and Astronomy, 2010. 53(10): p. 1805-1810.

46. Liu, L., et al., Improvement for sensitivity of biosensor with total internal reflection imaging ellipsometry (TIRIE). Thin Solid Films, 2011. 519(9): p. 2758-2762.

47. Zhang, Y.B., Y.Y. Chen, and G. Jin, Serum tumor marker detection on PEGylated lipid membrane using biosensor based on total internal reflection imaging ellipsometry. Sensors and Actuators B-Chemical, 2011. 159(1): p. 121-125. 
48. Kang, T., Y. Niu, and G. Jin, Visualization of the interaction between tris and lysozyme with a biosensor based on total internal reflection imaging ellipsometry. Thin Solid Films, 2014. 571: p. 463-467.

49. Liu, W., et al., Using Electrochemistry - Total Internal Reflection Ellipsometry Technique to Observe the Dissolved Oxygen Reduction on Clark Electrode. Electrochimica Acta, 2014. 142: p. 371-377.

50. Liu, W., et al., An Imaging Ellipsometry Approach to Dissolved Oxygen Measurement on Surface Tethered Weak Polyelectrolyte Modified Electrode. Journal of the Electrochemical Society, 2016. 163(5): p. H286-H291.

51. Kang, T.F., Y. Niu, and G. Jin, Visualization of the interaction between tris and lysozyme with a biosensor based on total internal reflection imaging ellipsometry. Thin Solid Films, 2014. 571: p. 463-467. 